ITP-UH-21/07

\title{
Scattering of Noncommutative Waves and Solitons in a Supersymmetric Chiral Model in 2+1 Dimensions
}

\author{
Christian Gutschwager*, Tatiana A. Ivanova ${ }^{\dagger}$ and Olaf Lechtenfeld* \\ *Institut für Theoretische Physik, Leibniz Universität Hannover \\ Appelstraße 2, 30167 Hannover, Germany \\ Email: gutschwager@math.uni-hannover.de, lechtenf@itp.uni-hannover.de \\ ${ }^{\dagger}$ Bogoliubov Laboratory of Theoretical Physics, JINR \\ 141980 Dubna, Moscow Region, Russia \\ Email: ita@theor.jinr.ru
}

\begin{abstract}
Interactions of noncommutative waves and solitons in $2+1$ dimensions can be analyzed exactly for a supersymmetric and integrable $\mathrm{U}(n)$ chiral model extending the Ward model. Using the Moyal-deformed dressing method in an antichiral superspace, we construct explicit timedependent solutions of its noncommutative field equations by iteratively solving linear equations. The approach is illustrated by presenting scattering configurations for two noncommutative $\mathrm{U}(2)$ plane waves and for two noncommutative $\mathrm{U}(2)$ solitons as well as by producing a noncommutative $\mathrm{U}(1)$ two-soliton bound state.
\end{abstract}




\section{Introduction and summary}

Solitonic solutions of the field equations of motion play an essential role in our understanding of field and string theories beyond perturbation theory. This persists for the noncommutative extension of scalar and supersymmetric gauge field theories which appear naturally in string theories [1]-[4]. In particular, the massless modes of the open $N=2$ string in a space-time filling brane with a constant NS $B$-field are described by noncommutative self-dual Yang-Mills (SDYM) theory in $2+2$ dimensions [5]. Upon reduction on the worldvolume of $n$ coincident D2-branes there emerges a noncommutative generalization [6] of a modified $\mathrm{U}(n)$ chiral model in $2+1$ dimensions known as the Ward model [7]. The integrability of this model [7]-[12] is preserved under the noncommutative deformation $[13,14]$. In [13]-[18] families of multi-soliton and plane-wave solutions to its Moyaldeformed equations of motions were studied. Also, reductions of wave configurations in the $2+1$ dimensional model to solutions of the noncommutative sine-Gordon equations were described [19]. We remark that not only the (noncommutative) Ward-model or sine-Gordon equations but a lot of other integrable equations in three and fewer dimensions derive from the (noncommutative) SDYM equations by suitable reductions (see e.g. [20]-[25] and references therein).

Given the fact that spacetime supersymmetry is an essential ingredient of string theory, it is natural to consider supersymmetric extensions of the above scenario. This was done by Witten [26] who has shown that $\mathcal{N}=4$ super SDYM theory appears in twistor string theory. ${ }^{1}$ Later, it was shown that $\mathcal{N}=4$ super SDYM in $2+2$ dimensions can be reduced to an $\mathcal{N}=8$ supersymmetric extension of the Ward model in $2+1$ dimensions [29]. Subsequently, truncations to $\mathcal{N}<8$ and a noncommutative Moyal deformation of this model were considered, and noncommutative multi-soliton solutions were constructed [30]. However, as in the non-supersymmetric case, generic multi-soliton configurations were found to be devoid of scattering [30] (see also [31]).

These supersymmetric no-scattering soliton configurations were obtained by applying a solutiongenerating technique (the dressing method [32]) to the $\mathcal{N}$-extended noncommutative $\mathrm{U}(n)$ Ward model, taking a dressing ansatz for the $\psi$ function with only first-order poles in the spectral parameter $\zeta$. Here we show that for multi-waves this ansatz yields nontrivial wave-wave interactions since each plane wave experiences a phase shift. Furthermore, by allowing for second-order poles in the dressing ansatz, we construct $\mathcal{N}$-extended noncommutative (time-dependent) two-soliton configurations with genuine soliton-soliton interaction. Thus, the studied features of the undeformed Ward model survive not only the Moyal deformation but the supersymmetric extension as well.

\section{The noncommutative $\mathcal{N}$-extended Ward model}

Recall that nonlinear sigma models in $2+1$ dimensions ${ }^{2}$ may be Lorentz-invariant or integrable but not both. An integrable model appears when one adds to the standard sigma-model field equations a Wess-Zumino-Witten term which explicitly breaks the Lorentz group $\mathrm{SO}(2,1)$ to the group $\mathrm{SO}(1,1) \cong \mathrm{GL}(1, \mathbb{R})[7]$. An $\mathcal{N}=8$ supersymmetric generalization of this model has been introduced in [29] and is easily truncated to any smaller even number $\mathcal{N}$ of supersymmetries. To formulate this model, one should introduce:

\footnotetext{
${ }^{1}$ For further developments and references see e.g. [27, 28].

${ }^{2}$ Sigma models in $k$ dimensions describe mappings of a $k$-dimensional manifold $X$ into a manifold $Y$. Chiral models pertain to the special case when $Y$ is a Lie group.
} 
- the space $\mathbb{R}^{2,1}=\left(\mathbb{R}^{3}, g\right)$ with coordinates $\left(x^{a}\right)=(t, x, y)$

- the metric $g=\operatorname{diag}(-1,+1,+1)$

- the superspace $\mathbb{R}^{3 \mid 2 \mathcal{N}}$ with coordinates $\left(x^{a} \mid \eta_{i}^{\alpha}, \theta^{i \alpha}\right)$ for $\alpha=1,2$ and $i=1, \ldots, \frac{1}{2} \mathcal{N} \leq 4$

- the antichiral superspace $\mathbb{R}^{3 \mid \mathcal{N}}$ with coordinates $\left(x^{a} \mid \eta_{i}^{\alpha}\right)$

The $\mathcal{N}$-extended Ward model describes the dynamics of a $\mathrm{U}(n)$-valued superfield $\Phi\left(x^{a}, \eta_{i}^{\alpha}\right)$ living on the antichiral superspace $\mathbb{R}^{3 \mid \mathcal{N}}$. The noncommutative Moyal deformation of this model was considered in [30].

Since integrability can be preserved in noncommutative deformations (see e.g. [13]-[19]), we right away Moyal deform the supersymmetric Ward model with a constant real noncommutativity parameter $\theta \geq 0$. This is achieved by replacing the ordinary product of classical fields (or their components) with the noncommutative associative star product, ${ }^{3}$

$$
(f \star g)\left(t, x, y, \eta_{i}^{\alpha}\right)=f\left(t, x, y, \eta_{i}^{\alpha}\right) \exp \left\{\frac{\mathrm{i}}{2} \theta\left(\overleftarrow{\partial_{x}} \overrightarrow{\partial_{y}}-\overleftarrow{\partial_{y}} \overrightarrow{\partial_{x}}\right)\right\} g\left(t, x, y, \eta_{i}^{\alpha}\right)
$$

Note that we choose a purely bosonic space-space deformation, i.e. the time coordinate remains commutative and no derivatives with respect to the Grassmann variables $\eta_{i}^{\alpha}$ appear in (2.1). The $\mathrm{U}(n)$-valued superfield $\Phi\left(t, x, y, \eta_{i}^{\alpha}\right)$ of the noncommutative $\mathcal{N}$-extended $\mathrm{U}(n)$ Ward model [30] obeys the classical field equations

$$
\begin{aligned}
& \partial_{x}\left(\Phi^{\dagger} \star \partial_{x} \Phi\right)+\partial_{y}\left(\Phi^{\dagger} \star \partial_{y} \Phi\right)-\partial_{t}\left(\Phi^{\dagger} \star \partial_{t} \Phi\right)+\partial_{y}\left(\Phi^{\dagger} \star \partial_{t} \Phi\right)-\partial_{t}\left(\Phi^{\dagger} \star \partial_{y} \Phi\right)=0, \\
& \partial_{1}^{i}\left(\Phi^{\dagger} \star \partial_{x} \Phi\right)-\partial_{t}\left(\Phi^{\dagger} \star \partial_{2}^{i} \Phi\right)+\partial_{y}\left(\Phi^{\dagger} \star \partial_{2}^{i} \Phi\right)=0, \\
& \partial_{1}^{i}\left(\Phi^{\dagger} \star \partial_{t} \Phi\right)+\partial_{1}^{i}\left(\Phi^{\dagger} \star \partial_{y} \Phi\right)-\partial_{x}\left(\Phi^{\dagger} \star \partial_{2}^{i} \Phi\right)=0, \\
& \partial_{1}^{i}\left(\Phi^{\dagger} \star \partial_{2}^{j} \Phi\right)+\partial_{1}^{j}\left(\Phi^{\dagger} \star \partial_{2}^{i} \Phi\right)=0,
\end{aligned}
$$

where $\partial_{\alpha}^{i}:=\partial / \partial \eta_{i}^{\alpha}$, and the unitarity condition reads $\Phi^{\dagger} \star \Phi=\Phi \star \Phi^{\dagger}=\mathbf{1}_{n}$, with $\dagger$ denoting hermitian conjugation. The Wess-Zumino-Witten terms responsible for the integrability are the last two terms in the first line above.

As it was discussed in $[29,30]$, the field equations (2.2) are equivalent to

$$
\hat{D}_{\alpha}^{i} \hat{\mathcal{A}}_{\beta}^{j}+\hat{D}_{\beta}^{j} \hat{\mathcal{A}}_{\alpha}^{i}+\hat{\mathcal{A}}_{\alpha}^{i} \star \hat{\mathcal{A}}_{\beta}^{j}+\hat{A}_{\beta}^{j} \star \hat{\mathcal{A}}_{\alpha}^{i}+(\alpha \leftrightarrow \beta)=0
$$

with the full superfields

$$
\hat{\mathcal{A}}_{1}^{i}=0 \quad \text { and } \quad \hat{\mathcal{A}}_{2}^{i}=\Phi^{\dagger} \star \hat{D}_{2}^{i} \Phi
$$

Here,

$$
\hat{D}_{\alpha}^{i}=\partial_{\alpha}^{i}+2 \theta^{i \beta} \partial_{(\alpha \beta)} \quad \text { with } \quad \partial_{(11)}=\partial_{t}-\partial_{y}, \partial_{(12)}=\partial_{(21)}=\partial_{x} \quad \text { and } \quad \partial_{(22)}=\partial_{t}+\partial_{y} .
$$

Furthermore, by expanding the superfields $\hat{\mathcal{A}}_{\alpha}^{i}$ in $\eta_{j}^{\beta}$, one can show that the equations (2.3) are equivalent to a supersymmetric extension of the Bogomolny-type Yang-Mills-Higgs equations on a multiplet $\left(A_{(\alpha \beta)}, \chi^{i \alpha}, H, \phi^{i j}, \tilde{\chi}_{i}^{\alpha}, G_{\alpha \beta}\right)$ of space-time component fields or its $\mathcal{N}<8$ truncation $[29,30]$. Here $A_{(\alpha \beta)}=A_{(\beta \alpha)}$ are the components of a vector in spinorial notation, $G_{\alpha \beta}=G_{\beta \alpha}$ are

\footnotetext{
${ }^{3}$ See [33] for reviews on noncommutative field theories.
} 
the components of a (pseudo)vector dual to a two-form, $\chi^{i \alpha}$ and $\tilde{\chi}_{i}^{\alpha}$ are components of spinors, and $\phi^{i j}=-\phi^{j i}$ are scalars in addition to the Higgs scalar $H$.

The supersymmetry algebra in $2+1$ dimensions is generated by the $2 \mathcal{N}$ supercharges

$$
\hat{Q}_{i \alpha}=\partial_{i \alpha}-2 \eta_{i}^{\beta} \partial_{(\alpha \beta)} \quad \text { and } \quad \hat{Q}_{\alpha}^{i}=\partial_{\alpha}^{i}
$$

The extended and deformed Ward-model equations (2.2) are invariant under the infinitesimal supersymmetry transformations generated by these supercharges because they anticommute with $\hat{D}_{\alpha}^{i}$.

In order to avoid cluttering the formulae we suppress the ' $\star$ ' notation for noncommutative multiplication from now on; all products are assumed to be star products, and functional operations (e.g. inverses) use the star product.

\section{Explicit solutions via the dressing approach}

Linear system. One of the powerful tools for constructing solutions to integrable equations is the so-called 'dressing method' [32] which is easily extended to the noncommutative and supersymmetric setup [13, 14, 30]. The key observation is that the field equations (2.3) (or (2.2)) can be obtained as compatibility conditions for a linear system of differential equations. The six antichiral superfield components

$$
\hat{\mathcal{A}}_{\alpha}^{i}=\mathcal{A}_{\alpha}^{i}+2 \theta^{i \beta}\left(\mathcal{A}_{(\alpha \beta)}-\varepsilon_{\alpha \beta} \mathcal{H}\right)
$$

defined by (2.4) and (2.5) read

$$
\begin{aligned}
& \mathcal{A}_{(12)}-\mathcal{H}=0 \quad \text { and } \quad \mathcal{A}_{(22)}=\Phi^{\dagger} \partial_{(22)} \Phi=: \mathcal{A} \\
& \mathcal{A}_{(11)}=0 \quad \text { and } \quad \mathcal{A}_{(12)}+\mathcal{H}=\Phi^{\dagger} \partial_{(12)} \Phi=: \mathcal{B} \\
& \mathcal{A}_{1}^{i}=0 \quad \text { and } \quad \mathcal{A}_{2}^{i}=\Phi^{\dagger} \partial_{2}^{i} \Phi \quad=: \mathcal{C}^{i}
\end{aligned}
$$

With these data, we consider the linear equations

$$
\begin{aligned}
\left(\zeta \partial_{x}-\partial_{t}-\partial_{y}\right) \psi & =\mathcal{A} \psi \\
\left(\zeta \partial_{t}-\zeta \partial_{y}-\partial_{x}\right) \psi & =\mathcal{B} \psi \\
\left(\zeta \partial_{1}^{i}-\partial_{2}^{i}\right) \psi & =\mathcal{C}^{i} \psi,
\end{aligned}
$$

where the $n \times n$ matrix $\psi$ depends on $\left(x^{a} \mid \zeta, \eta_{i}^{\alpha}\right)$ and the $n \times n$ matrices $\mathcal{A}, \mathcal{B}$ and $\mathcal{C}^{i}$ are superfield functions of $\left(x^{a} \mid \eta_{i}^{\alpha}\right) \in \mathbb{R}^{3 \mid \mathcal{N}}$ but do not depend on the spectral parameter $\zeta$ which lies in the extended complex plane $\mathbb{C} \cup\{\infty\}=\mathbb{C} P^{1}$.

Compatibility conditions. The compatibility conditions for the linear system (3.3) are

$$
\begin{aligned}
& \partial_{x} \mathcal{A}-\left(\partial_{t}+\partial_{y}\right) \mathcal{B}-[\mathcal{A}, \mathcal{B}]=0, \quad \partial_{2}^{i} \mathcal{A}-\left(\partial_{t}+\partial_{y}\right) \mathcal{C}^{i}+\left[\mathcal{C}^{i}, \mathcal{A}\right]=0 \\
& \partial_{2}^{i} \mathcal{B}-\partial_{x} \mathcal{C}^{i}+\left[\mathcal{C}^{i}, \mathcal{B}\right]=0, \quad\left\{\partial_{2}^{i}+\mathcal{C}^{i}, \partial_{2}^{j}+\mathcal{C}^{j}\right\}=0 \\
& \left(\partial_{t}-\partial_{y}\right) \mathcal{A}-\partial_{x} \mathcal{B}=0, \quad \partial_{1}^{i} \mathcal{B}-\left(\partial_{t}-\partial_{y}\right) \mathcal{C}^{i}=0 \\
& \partial_{1}^{i} \mathcal{A}-\partial_{x} \mathcal{C}^{i}=0, \quad \partial_{1}^{i} \mathcal{C}^{j}+\partial_{1}^{j} \mathcal{C}^{i}=0 .
\end{aligned}
$$


It is easy to see that (3.2) solves the first two lines and turns the last two lines into (2.2).

The $\operatorname{GL}(n, \mathbb{C})$-valued superfield $\psi$ is subject to the reality condition [30]

$$
\psi(t, x, y, \zeta, \eta)[\psi(t, x, y, \bar{\zeta}, \eta)]^{\dagger}=\mathbf{1}_{n} .
$$

Inserting the parametrization (3.2) of $\mathcal{A}, \mathcal{B}$ and $\mathcal{C}^{i}$ into the linear system (3.3), we obtain the standard gauge-fixing conditions

$$
\begin{aligned}
& \psi(t, x, y, \eta, \zeta \rightarrow \infty)=\mathbf{1}_{n}+O\left(\zeta^{-1}\right), \\
& \psi(t, x, y, \eta, \zeta \rightarrow 0)=\Phi^{\dagger}(t, x, y, \eta)+O(\zeta) .
\end{aligned}
$$

The second equation yields $\Phi=\psi^{-1}(\zeta=0)$ and also $\mathcal{A}, \mathcal{B}$ and $\mathcal{C}^{i}$ via (3.2).

Explicit $\mathcal{N}$-extended solutions. One can rewrite (3.3) in the form

$$
\psi\left(\partial_{t}+\partial_{y}-\zeta \partial_{x}\right) \psi^{\dagger}=\mathcal{A}, \quad \psi\left(\partial_{x}-\zeta \partial_{t}+\zeta \partial_{y}\right) \psi^{\dagger}=\mathcal{B} \quad \text { and } \quad \psi\left(\partial_{2}^{i}-\zeta \partial_{1}^{i}\right) \psi^{\dagger}=\mathcal{C}^{i},
$$

where the right-hand sides of (3.7) do not depend on $\zeta$. Therefore the left-hand sides of (3.7) as well as the reality condition (3.5) do not depend on $\zeta$, while $\psi$ is expected to be a nontrivial meromorphic function of $\zeta$ globally defined on $\mathbb{C} P^{1}$.

We briefly recall the dressing construction. We assume that $\psi$ possesses $m$ poles in $\zeta$ at mutually distinct locations $\mu_{k}$ for $k=1, \ldots, m$ in the complex lower half plane. One can build a solution $\psi_{m}$ featuring $m$ simple poles at $\mu_{1}, \ldots, \mu_{m}$ by left-multiplying an $(m-1)$-simple-pole solution $\psi_{m-1}$ with a single-pole factor of the form

$$
\mathbf{1}_{n}+\frac{\mu_{m}-\bar{\mu}_{m}}{\zeta-\mu_{m}} P_{m}\left(x^{a}, \eta_{i}^{\alpha}\right)
$$

Here, the $n \times n$ matrix function $P_{m}$ is a hermitian projector of rank $r_{m}$, i.e. $P_{m}^{\dagger}=P_{m}$ and $P_{m}^{2}=P_{m}$, and therefore one can decompose

$$
P_{m}=T_{m}\left(T_{m}^{\dagger} T_{m}\right)^{-1} T_{m}^{\dagger},
$$

where $T_{m}$ is an $n \times r_{m}$ matrix depending on $x^{a}$ and $\eta_{i}^{\alpha}$. So, the iteration $\psi_{1} \mapsto \ldots \mapsto \psi_{m}$ yields the multiplicative ansatz

$$
\psi_{m}=\prod_{\ell=0}^{m-1}\left(\mathbf{1}_{n}+\frac{\mu_{m-\ell}-\bar{\mu}_{m-\ell}}{\zeta-\mu_{m-\ell}} P_{m-\ell}\right)
$$

which, via a partial fraction decomposition, may be rewritten in the additive form

$$
\psi_{m}=\mathbf{1}_{n}+\sum_{k=1}^{m} \frac{\Lambda_{m k} S_{k}^{\dagger}}{\zeta-\mu_{k}}
$$

where $\Lambda_{m k}$ and $S_{k}$ are some $n \times r_{k}$ matrices depending on $x^{a}$ and $\eta_{i}^{\alpha}$.

In [30] it was shown that all Ward-model field equations are satisfied if one takes

$$
S_{k}=S_{k}\left(w_{k}, \eta_{k}^{i}\right) \quad \text { and } \quad T_{k}=\left\{\prod_{l=1}^{k-1}\left(\mathbf{1}_{n}-\frac{\mu_{k-l}-\bar{\mu}_{k-l}}{\mu_{k-l}-\bar{\mu}_{k}} P_{k-l}\right)\right\} S_{k}
$$


with

$$
w_{k}:=x+\frac{1}{2}\left(\bar{\mu}_{k}-\bar{\mu}_{k}^{-1}\right) y+\frac{1}{2}\left(\bar{\mu}_{k}+\bar{\mu}_{k}^{-1}\right) t \quad \text { and } \quad \eta_{k}^{i}:=\eta_{i}^{1}+\bar{\mu}_{k} \eta_{i}^{2} \quad \text { for } \quad k=1, \ldots, m .
$$

Substituting (3.12) into (3.9), we obtain from (3.10) the solution

$$
\Phi_{m}=\psi_{m}^{-1}(\zeta=0)=\prod_{k=1}^{m}\left(\mathbf{1}_{n}-\rho_{k} P_{k}\right) \quad \text { with } \quad \rho_{k}=1-\frac{\mu_{k}}{\bar{\mu}_{k}} .
$$

Furthermore, from (3.7) we read off

$$
\mathcal{A}=\sum_{k=1}^{m}\left(\mu_{k}-\bar{\mu}_{k}\right) \partial_{x} P_{k}, \quad \mathcal{B}=\sum_{k=1}^{m}\left(\mu_{k}-\bar{\mu}_{k}\right)\left(\partial_{t}-\partial_{y}\right) P_{k} \quad \text { and } \quad \mathcal{C}^{i}=\sum_{k=1}^{m}\left(\mu_{k}-\bar{\mu}_{k}\right) \partial_{1}^{i} P_{k}
$$

Thus, the solutions of the noncommutative $\mathcal{N}$-extended integrable $\mathrm{U}(n)$ chiral model in $2+1$ dimensions described by the simple-pole ansatz (3.10)-(3.12) are parametrized by the set $\left\{S_{k}\right\}_{1}^{m}$ of matrix-valued functions of $w_{k}$ and $\eta_{k}^{i}$ and by the pole positions $\mu_{k}$.

\section{Configuration of two noncommutative plane waves}

The solutions constructed in the previous section have solitonic character when all the functions $\left\{S_{1}\right\}_{1}^{m}$ are rational (see e.g. $[7,8,12,13,14,30]$ ). For the dressing ansatz (3.10) with pairwise distinct $\mu_{k}$ it was shown that no scattering occurs in the $\mathcal{N}$-extended $m$-soliton configuration [30]. However, it is known that in the bosonic commutative [10] and noncommutative [15, 17] cases the choice of exponentials for $\left\{S_{1}\right\}_{1}^{m}$ leads to a configuration of $m$ plane waves which do feature interaction. It is natural to expect that $\mathcal{N}$-extended plane-wave configurations have the same properties. We will demonstrate this on the example of a two-wave configuration which is a particular solution of the noncommutative $\mathcal{N}=2$ supersymmetric $\mathrm{U}(2)$ Ward model. Note that the properties of a solution describing $m$ extended waves essentially depends on $\mu_{k} \in \mathbb{C}$ and the parameters in $\left\{S_{k}\right\}_{1}^{m}$, and a complete study of the interaction of such waves is far from a trivial matter [10]. That is why we restrict ourselves to a special form of $S_{k}$ and a choice of parameters which simplifies the analysis.

Extended wave solution. Let us take $\mu$ to be purely imaginary,

$$
\mu=-\mathrm{i} p \quad \text { with } \quad p>1 .
$$

Then from (3.13) for $\mathcal{N}=2$ we obtain

$$
w=x+\frac{\mathrm{i}}{2 p}\left(\left(p^{2}+1\right) y+\left(p^{2}-1\right) t\right) \quad \text { and } \quad \eta=\eta^{1}+\mathrm{i} p \eta^{2} .
$$

We choose $T=S=S(w, \eta)$ in the form

$$
T=\left(\begin{array}{c}
1+\eta \varepsilon \\
\mathrm{e}^{b w}
\end{array}\right)
$$

where $\varepsilon$ is a Grassmann-odd parameter and $b=b^{x}+\mathrm{i} b^{y}$ is a complex number. The form (4.3) is obviously not the most general, but it extends the simplest bosonic wave ansatz by a nilpotent term. 
Our choice (4.1)-(4.3) then yields

$$
P=T\left(T^{\dagger} T\right)^{-1} T^{\dagger}=\left(\begin{array}{cc}
\frac{\bar{\alpha} \alpha}{\bar{\alpha} \alpha+\mathrm{e}^{2 \tilde{x}-\theta}} & \frac{\alpha \mathrm{e}^{\tilde{x}} \mathrm{e}^{-i \tilde{y}}}{\bar{\alpha} \alpha+\mathrm{e}^{2 \tilde{x}}} \\
\frac{\bar{\alpha} \mathrm{e}^{\tilde{x}} \mathrm{e}^{i \tilde{y}}}{\bar{\alpha} \alpha+\mathrm{e}^{2 \tilde{x}}} & \frac{\mathrm{e}^{2 \tilde{x}+\tilde{\theta}}}{\bar{\alpha} \alpha+\mathrm{e}^{2 \tilde{x}+\tilde{\theta}}}
\end{array}\right)
$$

with the abbreviations

$$
\begin{gathered}
\tilde{x}:=b^{x} x-\frac{b^{y}}{2 p}\left(\left(p^{2}+1\right) y+\left(p^{2}-1\right) t\right) \quad \text { and } \quad \tilde{y}:=b^{y} x+\frac{b^{x}}{2 p}\left(\left(p^{2}+1\right) y+\left(p^{2}-1\right) t\right) \\
\tilde{\theta}:=\frac{|b|^{2}}{2 p}\left(p^{2}+1\right) \theta, \quad \alpha:=1+\eta \varepsilon \quad \text { and } \quad \bar{\alpha}=1+\bar{\eta} \bar{\varepsilon}
\end{gathered}
$$

For the $\mathrm{U}(2)$-valued superfield $\Phi$ which is by construction a solution to (2.2), we finally find the one-wave configuration

$$
\Phi=\mathbf{1}_{n}-2 P=\left(\begin{array}{cc}
\frac{\mathrm{e}^{2 \tilde{x}-\tilde{\theta}}-\bar{\alpha} \alpha}{\mathrm{e}^{2 \tilde{x}-\theta}+\bar{\alpha} \alpha} & -\frac{2 \alpha \mathrm{e}^{\tilde{x}-i \tilde{y}}}{\mathrm{e}^{2 \tilde{x}}+\bar{\alpha} \alpha} \\
-\frac{2 \bar{\alpha} \mathrm{e}^{\tilde{x}+i \tilde{y}}}{\mathrm{e}^{2 \tilde{x}}+\bar{\alpha} \alpha} & -\frac{\mathrm{e}^{2 \tilde{x}+\tilde{\theta}}-\bar{\alpha} \alpha}{\mathrm{e}^{2 \tilde{x}+\tilde{\theta}}+\bar{\alpha} \alpha}
\end{array}\right)
$$

Notice that all expressions in (4.4) and (4.7) are formed with Moyal star multiplication.

The wave described by (4.3)-(4.7) simply moves at constant velocity which can be shown by the same arguments as in [10]. Moreover, the wave front lies along

$$
\tilde{x}=0
$$

which for fixed time is a straight line in the $x y$-plane. From (4.4) one can see that (cf. [17])

$$
\lim _{\tilde{x} \rightarrow-\infty} P=\left(\begin{array}{ll}
1 & 0 \\
0 & 0
\end{array}\right) \quad \text { and } \quad \lim _{\tilde{x} \rightarrow+\infty} P=\left(\begin{array}{ll}
0 & 0 \\
0 & 1
\end{array}\right)
$$

corresponding to the large-time limits $t \rightarrow \pm \infty$ for $b^{y}<0$ and finite $x, y{ }^{4}$ Thus, we get the asymptotics

$$
\Phi_{ \pm \infty}=\lim _{t \rightarrow \pm \infty} \Phi= \pm\left(\begin{array}{rr}
1 & 0 \\
0 & -1
\end{array}\right)
$$

which corresponds to straight wave moving far away from the $t=0$ line

$$
b^{x} x-\frac{b^{y}}{2 p}\left(p^{2}+1\right) y=0
$$

on either side.

Interacting waves. Now we consider two waves defined by formulae similar to (4.1)-(4.3). Namely, we choose

$$
\begin{aligned}
& \mu_{k}=-\mathrm{i} p_{k} \quad \text { and } \quad \eta_{k}=\eta^{1}+\mathrm{i} p_{k} \eta^{2} \quad \text { with } \quad p_{2}>p_{1}>1 \\
& w_{k}=x+\frac{\mathrm{i}}{2 p_{k}}\left(\left(p_{k}^{2}+1\right) y+\left(p_{k}^{2}-1\right) t\right) \quad \text { and } \quad \alpha_{k}=1+\eta_{k} \varepsilon_{k}
\end{aligned}
$$

\footnotetext{
${ }^{4}$ For $b^{y}>0$ we simply have the correspondence $\tilde{x} \rightarrow \pm \infty \Leftrightarrow t \rightarrow \mp \infty$.
} 


$$
S_{k}=\left(\begin{array}{c}
\alpha_{k} \\
\mathrm{e}^{b_{k} w_{k}}
\end{array}\right) \quad \text { for } \quad k=1,2
$$

where $\varepsilon_{k}$ are Grassmann-odd parameters and $b_{k}=b_{k}^{x}+\mathrm{i} b_{k}^{y}$ are complex numbers. We also introduce

$$
\tilde{x}_{k}:=b_{k}^{x} x-\frac{b_{k}^{y}}{2 p_{k}}\left(\left(p_{k}^{2}+1\right) y+\left(p_{k}^{2}-1\right) t\right) \quad \text { and } \quad \tilde{y}_{k}:=b_{k}^{y} x+\frac{b_{k}^{x}}{2 p_{k}}\left(\left(p_{k}^{2}+1\right) y+\left(p_{k}^{2}-1\right) t\right),
$$

so that

$$
b_{k} w_{k}=\tilde{x}_{k}+\mathrm{i} \tilde{y}_{k} \quad \text { and } \quad\left[\tilde{x}_{k}, \tilde{y}_{k}\right]=\mathrm{i} \tilde{\theta}_{k} \quad \text { with } \quad \tilde{\theta}_{k}:=\frac{\left|b_{k}\right|^{2}}{2 p_{k}}\left(p_{k}^{2}+1\right) \theta .
$$

The relations (3.12) read

$$
T_{1}=S_{1} \quad \text { and } \quad T_{2}=\left(\mathbf{1}_{2}-\frac{2 p_{1}}{p_{1}+p_{2}} P_{1}\right) S_{2}
$$

from which we construct the matrices

$$
P_{k}=T_{k}\left(T_{k}^{\dagger} T_{k}\right)^{-1} T_{k}^{\dagger} \quad \text { for } \quad k=1,2 \quad \text { and } \quad \Phi=\left(\mathbf{1}_{2}-2 P_{2}\right)\left(\mathbf{1}_{2}-2 P_{1}\right),
$$

arriving at a two-wave configuration.

Let us move with the second wave. This means that we consider points around its wave front defined by the equation

$$
\tilde{x}_{2} \equiv b_{2}^{x} x-\frac{b_{2}^{y}}{2 p_{2}}\left(\left(p_{2}^{2}+1\right) y+\left(p_{2}^{2}-1\right) t\right)=0,
$$

which is a line in the $x y$-plane moving in time. For a proper choice of parameters keeping $\tilde{x}_{2}$ finite while $\tilde{x}_{1} \rightarrow \pm \infty$, asymptotically the first wave will be far away from the second wave on either side. Specifically for $b_{1}^{y}<0,(4.18)$ and (4.9) give us

$$
\begin{aligned}
& \left.T_{2}\right|_{t, \tilde{x}_{1} \rightarrow-\infty} \rightarrow\left\{\mathbf{1}_{2}-\frac{2 p_{1}}{p_{1}+p_{2}}\left(\begin{array}{ll}
1 & 0 \\
0 & 0
\end{array}\right)\right\}\left(\begin{array}{c}
\alpha_{2} \\
\mathrm{e}^{b_{2} w_{2}}
\end{array}\right)=\mathrm{e}^{\gamma}\left(\begin{array}{c}
\alpha_{2} \\
\mathrm{e}^{b_{2} w_{2}-\gamma}
\end{array}\right), \\
& \left.T_{2}\right|_{t, \tilde{x}_{1} \rightarrow+\infty} \rightarrow\left\{\mathbf{1}_{2}-\frac{2 p_{1}}{p_{1}+p_{2}}\left(\begin{array}{ll}
0 & 0 \\
0 & 1
\end{array}\right)\right\}\left(\begin{array}{c}
\alpha_{2} \\
\mathrm{e}^{b_{2} w_{2}}
\end{array}\right)=\left(\begin{array}{c}
\alpha_{2} \\
\mathrm{e}^{b_{2} w_{2}+\gamma}
\end{array}\right),
\end{aligned}
$$

where

$$
\mathrm{e}^{\gamma}:=\frac{p_{2}-p_{1}}{p_{2}+p_{1}}
$$

As a consequence, we arrive at

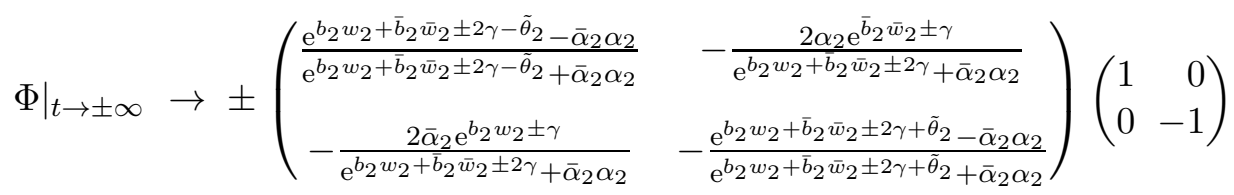

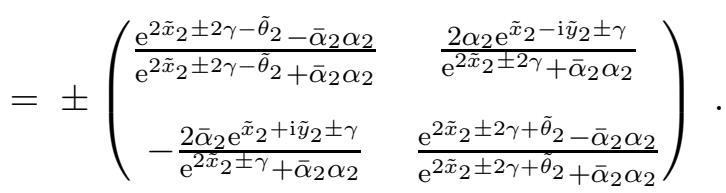


Comparing the $t \rightarrow \pm \infty$ limits of this configuration, we see that (up to sign) $\left.\Phi\right|_{t \rightarrow+\infty}$ deviates from $\left.\Phi\right|_{t \rightarrow-\infty}$ only by the phase shift $b_{2} w_{2}-\gamma \mapsto b_{2} w_{2}+\gamma$. This coincides with the $\mathcal{N}=0$ commutative result found in [10]. By symmetry, both waves experience the same phase shift of

$$
2 \gamma=2 \ln \frac{p_{2}-p_{1}}{p_{2}+p_{1}}
$$

Note that the explicit asymptotic form of $\Phi$ depends on $\theta$ but the phase shift does not. Although our two waves interact in a rather simple way, their dynamics depends essentially on the parameters $p_{k}, b_{k}$ and $\varepsilon_{k}$. The $\mathcal{N}=0$ waves are recovered by putting $\varepsilon_{k}=0$, i.e. $\alpha_{k}=1$. In the $\mathcal{N}$-extended case the choice of $S_{k}$ may be more general than (4.14), leading to more involved interaction dynamics.

\section{$5 \quad$ Two interacting solitons}

One-soliton configuration. According to the general formalism discussed in section 3, the time-dependent configuration for $m=1$ and $\mu$ not necessarily being purely imaginary simplifies to

$$
\psi=\mathbf{1}_{n}+\frac{\mu-\bar{\mu}}{\zeta-\mu} P \quad \text { and } \quad \Phi=\psi^{-1}(\zeta=0)=\mathbf{1}_{n}+\frac{\bar{\mu}-\mu}{\mu} P
$$

with

$$
P=T\left(T^{\dagger} T\right)^{-1} T^{\dagger} \quad \text { and } \quad T=T\left(w_{1}, \eta_{1}^{i}\right),
$$

where $w_{1}$ and $\eta_{1}^{i}$ are given in (3.13). This configuration will describe a moving soliton if the $n \times r$ matrix $T$ depends on $w_{1}$ rationally (cf. e.g. [7, 13, 30]). For $\mu=-\mathrm{i}$ we encounter the static case, where

$$
\psi=\mathbf{1}_{n}-\frac{2 \mathrm{i}}{\zeta+\mathrm{i}} P, \quad \Phi=\mathbf{1}_{n}-2 P, \quad w_{1}=z \equiv x+\mathrm{i} y \quad \text { and } \quad \eta_{1}^{i}=\eta^{i} \equiv \eta_{i}^{1}+\mathrm{i} \eta_{i}^{2}
$$

with $P$ and $T=T\left(w_{1}, \eta_{1}^{i}\right)=T\left(z, \eta^{i}\right)$ given in (5.2). The $n \times n$ matrix superfields $\mathcal{A}, \mathcal{B}$ and $\mathcal{C}^{i}$ from the linear system (3.3) and eqs. (3.4) are expressed in terms of $P$ as (cf. (3.15))

$$
\mathcal{A}=-2 \mathrm{i} \partial_{x} P, \quad \mathcal{B}=2 \mathrm{i} \partial_{y} P \quad \text { and } \quad \mathcal{C}^{i}=-2 \mathrm{i} \partial_{1}^{i} P
$$

We want to 'dress' the static solution (5.3) of the field equations (2.2) to produce a timedependent interacting two-soliton configuration of the Moyal-deformed supersymmetric Ward model. It is known in the non-supersymmetric case that soliton interactions appear only when higher-order poles in $\zeta$ are considered for the dressing ansatz $(3.10)[8,9,14]$. The simplest such situation occurs for a double pole at $\zeta=-\mathrm{i}$. Therefore, we take the static configuration given by (5.3) and (5.4) as our seed solution and consider the dressing transformation

$$
\psi \mapsto \tilde{\psi}=\left(\mathbf{1}_{n}-\frac{2 \mathrm{i}}{\zeta+\mathrm{i}} \tilde{P}\right) \psi=\left(\mathbf{1}_{n}-\frac{2 \mathrm{i}}{\zeta+\mathrm{i}} \tilde{P}\right)\left(\mathbf{1}_{n}-\frac{2 \mathrm{i}}{\zeta+\mathrm{i}} P\right)=\mathbf{1}_{n}-\frac{2 \mathrm{i}}{\zeta+\mathrm{i}}(P+\tilde{P})-\frac{4}{(\zeta+\mathrm{i})^{2}} \tilde{P} P .
$$

From the reality condition (3.5) we obtain the restrictions $\tilde{P}^{2}=\tilde{P}$ and $\tilde{P}^{\dagger}=\tilde{P}$, qualifying $\tilde{P}$ as a hermitian projector

$$
\tilde{P}=\tilde{T}\left(\tilde{T}^{\dagger} \tilde{T}\right)^{-1} \tilde{T}^{\dagger}
$$

built from some $n \times \tilde{r}$ matrix $\tilde{T}$. In the following we choose $r=1=\tilde{r}$, i.e. we consider rank one projectors $P$ and $\tilde{P}$. 
Demanding that $\tilde{\psi}$ is again a solution of the linear equations (3.3) with some new superfields $\tilde{\mathcal{A}}, \tilde{\mathcal{B}}$ and $\tilde{\mathcal{C}^{i}}$, we derive

$$
\begin{aligned}
& \tilde{\mathcal{A}}=\tilde{\psi}\left(\partial_{t}+\partial_{y}-\zeta \partial_{x}\right) \tilde{\psi}^{\dagger}=\left(\mathbf{1}_{n}-\frac{2 \mathrm{i}}{\zeta+\mathrm{i}} \tilde{P}\right) \mathcal{A}\left(\mathbf{1}_{n}+\frac{2 \mathrm{i}}{\zeta-\mathrm{i}} \tilde{P}\right)+\left(\mathbf{1}_{n}-\frac{2 \mathrm{i}}{\zeta+\mathrm{i}} \tilde{P}\right)\left(\partial_{t}+\partial_{y}-\zeta \partial_{x}\right)\left(\mathbf{1}_{n}+\frac{2 \mathrm{i}}{\zeta-\mathrm{i}} \tilde{P}\right), \\
& \tilde{\mathcal{B}}=\tilde{\psi}\left(\partial_{x}-\zeta \partial_{t}+\zeta \partial_{y}\right) \tilde{\psi}^{\dagger}=\left(\mathbf{1}_{n}-\frac{2 \mathrm{i}}{\zeta+\mathrm{i}} \tilde{P}\right) \mathcal{B}\left(\mathbf{1}_{n}+\frac{2 \mathrm{i}}{\zeta-\mathrm{i}} \tilde{P}\right)+\left(\mathbf{1}_{n}-\frac{2 \mathrm{i}}{\zeta+\mathrm{i}} \tilde{P}\right)\left(\partial_{x}-\zeta \partial_{t}+\zeta \partial_{y}\right)\left(\mathbf{1}_{n}+\frac{2 \mathrm{i}}{\zeta-\mathrm{i}} \tilde{P}\right), \\
& \tilde{\mathcal{C}}^{i}=\tilde{\psi}\left(\partial_{2}^{i}-\zeta \partial_{1}^{i}\right) \tilde{\psi}^{\dagger}=\left(\mathbf{1}_{n}-\frac{2 \mathrm{i}}{\zeta+\mathrm{i}} \tilde{P}\right) \mathcal{C}^{i}\left(\mathbf{1}_{n}+\frac{2 \mathrm{i}}{\zeta-\mathrm{i}} \tilde{P}\right)+\left(\mathbf{1}_{n}-\frac{2 \mathrm{i}}{\zeta+\mathrm{i}} \tilde{P}\right)\left(\partial_{2}^{i}-\zeta \partial_{1}^{i}\right)\left(\mathbf{1}_{n}+\frac{2 \mathrm{i}}{\zeta-\mathrm{i}} \tilde{P}\right) .
\end{aligned}
$$

The poles at $\zeta= \pm \mathrm{i}$ on the right-hand side of these equations have to be removable since $\tilde{\mathcal{A}}, \tilde{\mathcal{B}}$ and $\tilde{\mathcal{C}}^{i}$ are independent of $\zeta$. Putting to zero the corresponding residues, we find the conditions

$$
\begin{aligned}
& \left(\mathbf{1}_{n}-\tilde{P}\right)\left(\partial_{\bar{z}} \tilde{T}+\left(\partial_{\bar{z}} P\right) \tilde{T}\right)=0, \\
& \left(\mathbf{1}_{n}-\tilde{P}\right)\left(\partial_{t} \tilde{T}-2 \mathrm{i}\left(\partial_{z} P\right) \tilde{T}\right)=0, \\
& \left(\mathbf{1}_{n}-\tilde{P}\right)\left(\frac{1}{2}\left(\partial_{1}^{i}+\mathrm{i} \partial_{2}^{i}\right) \tilde{T}+\left(\partial_{1}^{i} P\right) \tilde{T}\right)=0 .
\end{aligned}
$$

After constructing a projector $\tilde{P}$ via a solution $\tilde{T}$ of these equations, we obtain a solution (5.5) of the linear equations (3.7) and, hence, a new (dressed) superfield

$$
\tilde{\Phi}=\tilde{\psi}^{-1}(\zeta=0)=\left(\mathbf{1}_{n}-2 P\right)\left(\mathbf{1}_{n}-2 \tilde{P}\right)
$$

obeying the field equations (2.2).

Explicit nonabelian solution. In order to generate an explicit example solving (5.8a)-(5.8c), we specialize to the group $\mathrm{U}(2)$ (i.e. choose $n=2$ ) and take as a one-soliton seed configuration

$$
P=T\left(T^{\dagger} T\right)^{-1} T^{\dagger} \quad \text { with } \quad T=\left(\begin{array}{c}
1 \\
f\left(z, \eta^{i}\right)
\end{array}\right),
$$

where implicit $\star$ products are still assumed everywhere. Inspired by the known form of $\tilde{T}$ in the bosonic case $[8,14]$, we make the ansatz

$$
\tilde{T}=T+T_{\perp}\left(T_{\perp}^{\dagger} T_{\perp}\right)^{-1} g \quad \text { with } \quad T_{\perp}=\left(\begin{array}{c}
\overline{f\left(z, \eta^{i}\right)} \\
-1
\end{array}\right)
$$

being orthogonal to $T$, i.e.

$$
T^{\dagger} T_{\perp}=0 \quad \Rightarrow \quad P T_{\perp}=0 \quad \text { and } \quad \mathbf{1}_{2}-P=T_{\perp}\left(T_{\perp}^{\dagger} T_{\perp}\right)^{-1} T_{\perp}^{\dagger},
$$

and with $g\left(t, z, \bar{z}, \eta^{i}, \bar{\eta}^{i}\right)$ being a superfield to be determined.

Substituting (5.11) into (5.8a), we get

$$
\partial_{\bar{z}} g=0 \quad \Rightarrow \quad g=g\left(t, z, \eta^{i}, \bar{\eta}^{i}\right) .
$$

From (5.8b) it follows that

$$
\partial_{t} g=-2 \mathrm{i} \partial_{z} f \quad \Rightarrow \quad g=-2 \mathrm{i}\left(t \partial_{z} f+H\left(z, \eta^{i}, \bar{\eta}^{i}\right)\right)
$$


Finally, from (5.8c) we obtain

$$
\bar{\partial}_{i} g=-\partial_{i} f \quad \Rightarrow \quad g=-2 \mathrm{i}\left(t \partial_{z} f+h\left(z, \eta^{i}\right)\right)+\bar{\eta}^{i} \partial_{i} f,
$$

where $h\left(z, \eta^{i}\right)$ is an arbitrary function of $z$ and $\eta^{i}$, and we have used the abbreviations (cf. (5.3))

$$
\partial_{i}:=\frac{1}{2}\left(\partial_{1}^{i}-\mathrm{i} \partial_{2}^{i}\right) \quad \text { and } \quad \bar{\partial}_{i}:=\frac{1}{2}\left(\partial_{1}^{i}+\mathrm{i} \partial_{2}^{i}\right)
$$

For further analysis we expand $f$ and $h$ in $\eta^{i}$,

$$
f\left(z, \eta^{i}\right)=f_{0}(z)+\eta^{i} f_{i}(z)+\ldots \quad \text { and } \quad h\left(z, \eta^{i}\right)=h_{0}(z)+\eta^{i} h_{i}(z)+\ldots .
$$

If we restrict ourselves to a bosonic subsector, studied in $[8,14]$, then the choice

$$
f_{0}=z \quad \text { and } \quad h_{0}=z^{2}
$$

yields a configuration of two lumps centered at $z= \pm \sqrt{-t}$, which for negative times accelerate symmetrically along the $x$-axis towards the origin $z=0$ of the Moyal plane, interact at small $t$, and decelerate to infinity along the $y$-axis for positive times. Thus, a head-on collision of these lumps results in a $90^{\circ}$ scattering. For the general superfield solution given by (5.9)-(5.11) and (5.15), the analysis seems much more complicated even when $\mathcal{N}=2$. However, for any $\mathcal{N} \leq 8$ and $f_{0}, h_{0}$ chosen as above, the bosonic core of the solution behaves in the above-described way. Hence, two $\mathcal{N}$-extended lumps carrying fermionic degrees of freedom can interact in the Moyal plane. We postpone a full-fledged scattering analysis of these supersymmetric configurations to future work.

Explicit abelian solution. A genuinely novel feature of noncommutative sigma models is the appearance of abelian solitons, i.e. nontrivial solutions for the group U(1). To describe these, one must employ the Moyal-Weyl correspondence and represent the noncommutativity by operatorvalued functions of only $\left(t \mid \eta_{i}^{\alpha}, \theta^{i \alpha}\right)$ instead of $\mathbb{C}$-number functions of $\left(t, z, \bar{z} \mid \eta_{i}^{\alpha}, \theta^{i \alpha}\right)$ subject to $\star$ multiplication. These operators act on an auxiliary Fock space $\mathcal{F}$ spanned by the basis

$$
|\ell\rangle=\frac{\left(a^{\dagger}\right)^{\ell}}{\sqrt{\ell !}}|0\rangle \quad \text { for } \quad \ell=0,1,2, \ldots \quad \text { and } \quad a|0\rangle=0 \quad \text { where } \quad\left[a, a^{\dagger}\right]=1 .
$$

Here, $\sqrt{2 \theta} a$ is the operator corresponding to the coordinate function $z$, and likewise for the hermitian conjugate. In this setting, projectors of finite rank $r$ in the total space $\mathbb{C}^{n} \otimes \mathcal{F}$ decompose as

$$
P=|T\rangle\langle T \mid T\rangle^{-1}\langle T|, \quad \text { where }|T\rangle=\left(\left|T_{1}\right\rangle,\left|T_{2}\right\rangle, \ldots,\left|T_{r}\right\rangle\right)
$$

denotes a row of $r$ kets from $\mathbb{C}^{n} \otimes \mathcal{F}$. In the following we take $n=1$ and $r=1$ (and drop the index).

It was demonstrated in [30] that the U(1) solutions are based on a coherent state

$$
|T\rangle=\left|\alpha\left(\eta^{i}\right)\right\rangle=\mathrm{e}^{\alpha\left(\eta^{i}\right) a^{\dagger}}|0\rangle
$$

with a Grassmann-valued parameter $\alpha$. We may always translate a static soliton to the origin of the Moyal plane, which amounts to dropping the body part of $\alpha$. Considering $\mathcal{N}=2$ supersymmetry, i.e. a single complex Grassmann-odd coordinate $\eta$, this implies

$$
\alpha(\eta)=\eta \epsilon
$$


with a Grassmann-odd parameter $\epsilon$, and thus

$$
|T\rangle=|\eta \epsilon\rangle=|0\rangle+\eta \epsilon|1\rangle .
$$

The corresponding projector is easily computed, and the final static abelian rank-one one-soliton configuration reads (in $\star$-product formulation)

$$
\Phi=1-4 \mathrm{e}^{-|z|^{2} / \theta}\left\{1+\frac{2 \bar{z}}{\sqrt{2 \theta}} \eta \epsilon+\frac{2 z}{\sqrt{2 \theta}} \bar{\epsilon} \bar{\eta}+2\left(\frac{|z|^{2}}{\theta}-1\right) \epsilon \bar{\epsilon} \eta \bar{\eta}\right\} .
$$

To construct a time-dependent abelian two-soliton solution (5.9), we must dress the seed solution based on (5.23) with a factor of $1-2 \tilde{P}$ based on a second ket $|\tilde{T}\rangle$. Again taking the rank $\tilde{r}=1$, we solve (5.8a)-(5.8c) and find

$$
|\tilde{T}\rangle=|T\rangle+\left|T_{\perp}^{1}\right\rangle g_{1}(t)+\left|T_{\perp}^{2}\right\rangle g_{2}(t) \quad \text { with } \quad\left\langle T \mid T_{\perp}^{1,2}\right\rangle=0
$$

where $g_{1}(t)=1$ and $g_{2}(t)=-\mathrm{i} t \sqrt{2 / \theta}$ multiply the kets

$$
\left|T_{\perp}^{1}\right\rangle=(\eta \bar{\epsilon}+\epsilon \bar{\eta})|1\rangle \quad \text { and } \quad\left|T_{\perp}^{2}\right\rangle=\bar{\eta} \bar{\epsilon}|0\rangle+(1-\epsilon \bar{\epsilon} \eta \bar{\eta})|1\rangle+\sqrt{2} \eta \epsilon|2\rangle
$$

respectively. As in the bosonic case, the time dependence drops out for $t \rightarrow \pm \infty$, and the two limits yield the same asymptotic configuration, which is supported near the origin. Hence, this configuration describes a two-soliton bound state dressed with a fermionic degree of freedom.

\section{Acknowledgements}

The authors are grateful to A.D. Popov for fruitful discussions and useful comments. O.L. thanks P. Aschieri, P. Kulish and J. Madore for discussions. T.A.I. acknowledges the Heisenberg-Landau program and the Russian Foundation for Basic Research (grant 06-01-00627-a) for partial support

and the Institut für Theoretische Physik der Leibniz Universität Hannover for its hospitality. The work of O.L. is partially supported by the Deutsche Forschungsgemeinschaft.

\section{References}

[1] M.R. Douglas and C.M. Hull, J. High Energy Phys. 02 (1998) 008 [hep-th/9711165].

[2] C.S. Chu and P.M. Ho, Nucl. Phys. B 550 (1999) 151 [hep-th/9812219].

[3] V. Schomerus, J. High Energy Phys. 06 (1999) 030 [hep-th/9903205].

[4] N. Seiberg and E. Witten, J. High Energy Phys. 09 (1999) 032 [hep-th/9908142].

[5] O. Lechtenfeld, A.D. Popov and B. Spendig, Phys. Lett. B 507 (2001) 317 [hep-th/0012200].

[6] O. Lechtenfeld, A.D. Popov and B. Spendig, J. High Energy Phys. 06 (2001) 011 [hep-th/0103196].

[7] R.S. Ward, J. Math. Phys. 29 (1988) 386; Commun. Math. Phys. 128 (1990) 319. 
[8] R.S. Ward, Phys. Lett. A 208 (1995) 203;

T.A. Ioannidou, J. Math. Phys. 37 (1996) 3422 [hep-th/9604126].

[9] T.A. Ioannidou and W.J. Zakrzewski, J. Math. Phys. 39 (1998) 2693 [hep-th/9802122].

[10] R. Leese, J. Math. Phys. 30 (1989) 2072.

[11] T. Ioannidou and N.S. Manton, Proc. Roy. Soc. Lond. A 461 (2005) 1965 [hep-th/0409168];

M. Dunajski and N.S. Manton, Nonlinearity 18 (2005) 1677 [hep-th/0411068].

[12] B. Dai and C.L. Terng,

"Backlund transformations, Ward solitons, and unitons", math.DG/0405363;

B. Dai, C.L. Terng and K. Uhlenbeck,

"On the space-time monopole equation", math.DG/0602607.

[13] O. Lechtenfeld and A.D. Popov, J. High Energy Phys. 11 (2001) 040 [hep-th/0106213].

[14] O. Lechtenfeld and A.D. Popov, Phys. Lett. B 523 (2001) 178 [hep-th/0108118].

[15] S. Bieling, J. Phys. A 35 (2002) 6281 [hep-th/0203269].

[16] M. Wolf, J. High Energy Phys. 06 (2002) 055 [hep-th/0204185].

[17] M. Ihl and S. Uhlmann, Int. J. Mod. Phys. A 18 (2003) 4889 [hep-th/0211263].

[18] M. Klawunn, O. Lechtenfeld and S. Petersen,

J. High Energy Phys. 06 (2006) 028 [hep-th/0604219].

[19] O. Lechtenfeld, L. Mazzanti, S. Penati, A.D. Popov and L. Tamassia, Nucl. Phys. B 705 (2005) 477 [hep-th/0406065].

[20] R.S. Ward, Phil. Trans. Roy. Soc. Lond. A 315 (1985) 451; "Multidimensional integrable systems," In: Field Theory, Quantum Gravity and Strings, Eds. H.J. De Vega, N. Sanchez, Vol. 2, p.106, 1986.

[21] L.J. Mason and G.A.J. Sparling, Phys. Lett. A 137 (1989) 29; J. Geom. Phys. 8 (1992) 243; L.J. Mason and M.A. Singer, Commun. Math. Phys. 166 (1994) 191.

[22] S. Chakravarty, M.J. Ablowitz and P.A. Clarkson, Phys. Rev. Lett. 65 (1990) 1085; M.J. Ablowitz, S. Chakravarty and L.A. Takhtajan, Commun. Math. Phys. 158 (1993) 289;

S. Chakravarty, S.L. Kent and E.T. Newman, J. Math. Phys. 36 (1995) 763;

M.J. Ablowitz, S. Chakravarty and R.G. Halburd, J. Math. Phys. 44 (2003) 3147.

[23] T.A. Ivanova and A.D. Popov, Phys. Lett. A 170 (1992) 293;

Phys. Lett. A 205 (1995) 158 [hep-th/9508129]; Theor. Math. Phys. 102 (1995) 280;

M. Legaré and A. D. Popov, JETP Lett. 59 (1994) 883; Phys. Lett. A 198 (1995) 195.

[24] M. Hamanaka and K. Toda, Phys. Lett. A 316 (2003) 77 [hep-th/0211148];

J. Phys. A 36 (2003) 11981 [hep-th/0301213];

M. Hamanaka, Phys. Lett. B 625 (2005) 324 [hep-th/0507112]; 
[25] A. Dimakis and F. Mueller-Hoissen, J. Phys. A 33 (2000) 6579 [nlin.si/0006029];

M.T. Grisaru, L. Mazzanti, S. Penati and L. Tamassia, J. High Energy Phys. 04 (2004) 057 [hep-th/0310214];

J. High Energy Phys. 04 (2004) 057 [hep-th/0310214];

I. Cabrera-Carnero, J. High Energy Phys. 10 (2005) 071 [hep-th/0503147].

[26] E. Witten, Commun. Math. Phys. 252 (2004) 189 [hep-th/0312171].

[27] A.D. Popov and C. Saemann, Adv. Theor. Math. Phys. 9 (2005) 931 [hep-th/0405123];

O. Lechtenfeld and C. Saemann, J. High Energy Phys. 03 (2006) 002 [hep-th/0511130];

A.D. Popov and M. Wolf, Commun. Math. Phys. 275 (2007) 685 [hep-th/0608225].

[28] J. Bedford, C. Papageorgakis and K. Zoubos,

"Twistor Strings with Flavour," arXiv:0708.1248 [hep-th].

[29] A.D. Popov, Phys. Lett. B 647 (2007) 509 [hep-th/0702106].

[30] O. Lechtenfeld and A.D. Popov, J. High Energy Phys. 06 (2007) 065 [arXiv:0704.0530 [hep-th]]

[31] M. Hamanaka, Y. Imaizumi and N. Ohta, Phys. Lett. B 529 (2002) 163 [hep-th/0112050].

[32] V.E. Zakharov and A.V. Mikhailov, Sov. Phys. JETP 47 (1978) 1017;

V.E. Zakharov and A.B. Shabat, Funct. Anal. Appl. 13 (1979) 166;

P. Forgács, Z. Horváth and L. Palla, Nucl. Phys. B 229 (1983) 77;

K. Uhlenbeck, J. Diff. Geom. 30 (1989) 1;

O. Babelon and D. Bernard, Commun. Math. Phys. 149 (1992) 279 [hep-th/9111036].

[33] A. Konechny and A.S. Schwarz, Phys. Rept. 360 (2002) 353 [hep-th/0012145, hep-th/0107251];

M.R. Douglas and N.A. Nekrasov, Rev. Mod. Phys. 73 (2001) 977 [hep-th/0106048];

R.J. Szabo, Phys. Rept. 378 (2003) 207 [hep-th/0109162]. 